\title{
Transcriptomic responses of the aphid Myzus persicae nicotianae Blackman (Hemiptera: Aphididae) to insecticides: Analyses in the single Chilean clone of the tobacco aphid
}

\author{
Marco Cabrera-Brandt ${ }^{1}$,Andrea X.Silva ${ }^{1}$, Gaël Le Trionnaire $^{2}$, Denis Tagu², and Christian C.Figueroa ${ }^{3 *}$
}

The tobacco aphid Myzus persicae nicotianae Blackman is a subspecies of the highly polyphagous and agricultural pest Myzus persicae (Sulzer). For its control, insecticide applications are widely used, but resistance to numerous molecules has been reported, displaying at least three insecticide resistance mechanisms, including: (i) elevated carboxylesterases (E-Carb), (ii) modification of the acetylcholinesterase (MACE), and (iii) $k d r$ and super- $k d r$ insensitivity mutations. In Chile, populations of the tobacco aphid are characterized by the presence of a single predominant clone, which is also present in high proportions in other countries of the Americas. This aphid clone exhibits low levels of carboxylesterase activity and is $k d r$ susceptible, but the MACE mechanism of insecticide resistance has not been studied. In order to characterize the tobacco aphid in terms of the MACE mechanism and to identify a preliminary group of aphid genes putatively involved in insecticide resistance, a cDNA microarray was used to study the transcriptomic responses when aphids are sprayed with a carbamate insecticide. The single Chilean clone of the tobacco aphid was characterized as MACE susceptible, but we found 38 transcripts significantly regulated by insecticide exposure (13 up- and 25 down-regulated genes). The expression of six of them was validated by qRT-PCR experiments at several time points $(6,12,18,24,30$, 36, and $42 \mathrm{~h})$ after insecticide application. This mutational and transcriptomic characterization of the tobacco aphid responding to insecticide spray opens new hypotheses in the understanding of the molecular mechanisms underlying insecticide resistance.

Key words: Insecticide resistance, MACE mechanism, Myzus persicae, transcriptomic analysis.

\section{INTRODUCTION}

Since the apparition of the first synthetic insecticide (1,1,1-trichloro-2,2-bis(4-chlorophenyl)ethane, DDT) in 1939, human efforts have been directed to lessen the resistance that sooner or later, most arthropod species develop (Yu, 2008). In fact, the systematic use of a wide array of insecticides, with variable dosage and at various frequencies of applications, represents a broad range of selective regimes on insect pest populations. This scenario can ultimately lead to the appearance of resistance to multiple insecticide families and concentrations, causing an army race between insects and humans that have caused a number of non-desirable effects such as decreased crop yields, accumulation of pesticide residues in foods, negative environmental impacts on

\footnotetext{
${ }^{1}$ Universidad Austral de Chile, Facultad de Ciencias, Campus Isla Teja, Casilla 567, Valdivia, Chile.

${ }^{2}$ Institut National de la Recherche Agronomique (INRA), Institut de Génétique, Environnement et Protection des Plantes (IGEPP), F-35653 Le Rheu, France.

${ }^{3}$ Universidad de Talca, Instituto de Ciencias Biológicas, 2 Norte 685, Talca, Chile. *Corresponding author (alfigueroa@utalca.cl).

Received: 4 June 2013.

Accepted: 25 March 2014.

doi:10.4067/S0718-58392014000200010
}

beneficial insects, and increased pollution in air, soil and water (Chowdhury et al., 2012). Most insecticides affect the insect nervous system, resulting in uncontrollable muscular contractions and lactic acidosis (Yu, 2008). So far, two main mechanisms that confer insecticide resistance in insects have been reported: (i) insensitivity resistance, referred to a modification of insecticide targetsites that reduce or abolish insecticide sensitivity, and (ii) metabolic resistance through increased activities of detoxifying enzymes (Ffrench-Constant et al., 2004).

The study of the molecular mechanisms involved in insecticide resistance began in the early 90's, revealing enzymatic mechanisms of sequestration and degradation of insecticides (Raymond et al., 1998) and target site mutations (Li et al., 2007). Recent advances in genomics and post-genomics allowed significant progress in the field of insecticide resistance by especially facilitating the study of more complex metabolic pathways and greatly expanding our views on the range of options available for insects to develop insecticide resistance (FfrenchConstant et al., 2004).

Aphids (Hemiptera: Aphididae) are herbivorous insects distributed worldwide that feed exclusively from phloem sap. Aphids are important pests of main cultivated crops causing significant economical loses due to direct damages by feeding and indirect damages 
by transmitting phytoviruses and contributing to fungus proliferation (Blackman and Eastop, 2000; Moury et al., 2007). Systematic insecticide applications are used to control aphid populations, but resistance to a wide range of insecticides (i.e., organophosphates, carbamates, pyrethroids, organochlorines, and neonicotinoids) has been reported in approximately 20 aphid species (van-Emden and Harrington, 2007). Among aphids, Myzus persicae (Sulzer) (Hemiptera: Aphididae) is a cosmopolitan and highly polyphagous pest that can provoke significant economic impacts (Blackman and Eastop, 2000; vanEmden and Harrington, 2007). Myzus persicae nicotianae is a subspecies of $M$. persicae, particularly well adapted to tobacco (Nicotiana tabacum L.) (Solanaceae) (Blackman and Eastop, 2000; Margaritopoulos et al., 2000). This tobacco aphid has developed at least four mechanisms of insecticide resistance, which are also found in M. persicae, including: (i) elevated carboxylesterases (E-Carb) levels, which confers resistance to organophosphates, carbamates and pyrethroids (Criniti et al., 2008), (ii) modification of the acetylcholinesterase (MACE), which confers resistance to organophosphates and carbamates (Anstead et al., 2008), (iii) $k d r$ and super-kdr mutations in the voltage-gated sodium channel, which confers resistance to pyrethroids and organochlorines (Criniti et al., 2008), and (iv) a mutation of a nicotinic acetylcholine receptor $\beta$ subunit that is associated with resistance to neonicotinoid insecticides (Bass et al., 2011). More recently, a new metabolic mechanism based on the amplification of a cytochrome P450 monooxygenase gene has been reported in $M$. persicae sampled on tobacco, which confers resistance to neonicotinoids, the most prominent class of insecticides currently used in the field (Puinean et al., 2010).

In Chile, the tobacco aphid populations are characterized by the presence of one single predominant clone (Fuentes-Contreras et al., 2004), which is also widespread in USA, Brazil, and Argentina (ZepedaPaulo et al., 2010), being considered as a "superclone" (a predominant and time persisting aphid clone characterized by its ecological success in terms of geographic distribution and abundance) (Vorburger et al., 2003). This tobacco aphid clone in Chile exhibits low levels of carboxylesterase activity, being classified as R1 (moderately resistant) and does not exhibit the mutation in the sodium channel (i.e. susceptible $k d r$ ) (FuentesContreras et al., 2004), but the MACE mechanism of insecticide resistance has not been studied yet. Despite the apparent insecticide susceptibility exhibited by this aphid clone, its large ecological success is intriguing considering its high frequency in populations and its wide distribution range in The Americas (Zepeda-Paulo et al., 2010), where insecticides such as neonicotinoid, pyrethroids, organophosphates, and carbamates are commonly used (Fuentes-Contreras, 2013, Universidad de Talca, personal communication).
This work aims at characterizing the tobacco aphid in Chile in terms of the MACE mechanism of insecticide resistance and identifying a preliminary group of RNAs differentially expressed in response to insecticide spray. To search for those genes, a heterologous cDNA microarray containing around 7000 transcripts of the pea aphid (Acyrthosiphon pisum, Harris, 1776) or the green-peach aphid (Myzus persicae) was used to study the transcriptomic response of tobacco aphids exposed to Pirimor (pirimicarb, 2-dimethylamino-5,6dimethylpyrimidin-4-yl dimethylcarbamate), a carbamate insecticide. Resulting in MACE insecticide susceptibility, the Chilean superclone of the tobacco aphid significantly regulated 38 transcripts by insecticide application. The expression of six of those genes was further studied at several time points after insecticide application using qRT-PCR. Putative metabolic insecticide resistance mechanisms are discussed.

\section{MATERIALS AND METHODS}

\section{Aphid rearing}

Aphids were individually reared on N. tabacum var. Burley 'BY64' (Profigen do Brazil, Brazil) under controlled conditions $\left(20 \pm 0.1{ }^{\circ} \mathrm{C} ; 16: 8 \mathrm{~h}\right.$ photoperiod) to ensure the parthenogenetic reproduction of aphids. All the experimental aphid lineages were genotyped with seven microsatellite loci to verify its genetic identity (i.e., they belong to the reported Chilean tobacco aphid superclone) (Fuentes-Contreras et al., 2004; Zepeda-Paulo et al., 2010). Only wingless aphids were used.

\section{MACE mutation identification}

Five individual aphids were used to characterize the MACE mutation (S431F) (Nabeshima et al., 2003) using quantitative PCR, as described in Anstead et al. (2008). The test consists in an allelic discrimination using the TaqMan PCR assay (Livak, 1999) and requires two specific primers to amplify a section of the DNAcontaining the mutation (Silva et al., 2012). Two reporter probes differentially labeled with VIC and FAM dyes were used to detect DNA fragments containing the MACE mutation. During each PCR cycle, the probes hybridize with their complimentary PCR products, which ultimately results in an increase in one or two fluorescent products, according to allelic distribution (i.e., heterozygous or homozygous, respectively).

\section{Insecticide bioassays}

For the preliminary identification of putatively regulated genes using cDNA microarrays, 20 synchronized wingless adult individuals of the Chilean tobacco aphid superclone were placed on tobacco foliar discs and sprayed with $1 \mathrm{~mL}$ at $20 \mathrm{mg} \mathrm{L}^{-1}\left(\mathrm{LC}_{50}\right.$ previously determined by the Probit method) of the commercial carbamate insecticide Pirimor (Syngenta) dissolved in water using a Potter Tower. 
Another batch of aphids on foliar discs was sprayed only with $\mathrm{H}_{2} \mathrm{O}$ as a control. After $12 \mathrm{~h}$ of application, all living aphids were removed and immediately frozen in liquid nitrogen for subsequent RNA isolation. This experiment was repeated three-times in order to obtain three independent biological replicates per condition.

For the quantification of the transcripts found as regulated in the microarray experiment, another two independent biological replicates were prepared following the same protocol described above. To improve the accuracy and reliability of the expression profiles, however, the commercial insecticide Pirimor was replaced by the pure active ingredient 2-dimethylamino5,6-dimethylpyrimidin-4-yl dimethylcarbamate. For each biological replicate, 300 synchronized wingless adult aphids were placed in groups of 20 individuals, and sprayed either with the pure insecticide or water as a control. After $6,12,18,24,30,36$, and $42 \mathrm{~h}$ of spraying, living aphids were removed and frozen in liquid nitrogen for subsequent RNA isolation.

\section{RNA isolation and cDNA preparation}

Total RNAs were extracted from whole bodies of treated (T) and control (C) aphids using the RNeasy Plant Mini kit (Qiagen, Germantown, Maryland, USA). The RNAs were resuspended in RNase-free water, and the RNAs integrity checked using a Bioanalyzer 2100 (Agilent Technologies, Santa Clara, California, USA). The T and $\mathrm{C}$ samples were then split into two technical replicates of $1 \mu \mathrm{g}$ total RNA. For these four samples (T1, T2; C1, C2), the mRNAs were amplified using MessageAmp aRNA kit (Ambion, Austin, Texas, USA), and the RNA quality checked again. From each technical replicate, $5 \mu \mathrm{g}$ of aRNA were used as template for cDNA synthesis. The cDNAs were then labeled with $\mathrm{Cy} 3$ and Cy5 dyes using ChipShot TM Indirect Labeling and Clean UP system (Promega, Madison, Wisconsin, USA), and purified using the CyScribe GFX Purification kit (Amersham Biosciences-GE, Fairfield, Connecticut, USA).

\section{Microarray hybridizations}

The cDNA microarray used for hybridizations contained probes for 7166 aphid genes (6776 ESTs cloned from Acyrthosiphon pisum and 390 from Myzus persicae), as described in Le Trionnaire et al. (2009). Pre-hybridization was performed at $42^{\circ} \mathrm{C}$ for $1 \mathrm{~h}$ in $2 \mathrm{~mL}$ ChipSpread buffer containing $4 \mathrm{x} \mathrm{SSC}(\mathrm{NaCl}$, sodium citrate) and $0.2 \%$ SDS. Hybridizations were then performed using the labeled cDNA T1, T2, C1, and C2 samples. Each treated sample was mixed with control samples (T1Cy3 vs. C1Cy5; T1Cy5 vs. С1Сy3; Т2Сy3 vs. С2Cy5, and Т2Cy5 vs. $\mathrm{C} 2 \mathrm{Cy} 3)$ with opposite dye swap to take into account the dye incorporation variability. This resulted in four hybridizations performed at $42{ }^{\circ} \mathrm{C}$ during $6 \mathrm{~h}$ (Ventana Medical Systems, Tucson, Arizona, USA) on a Discovery XT System hybridization robot with the ChipMap
80 kit (Ventana Medical Systems) at INRA-SCRIBE transcriptomic facilities (IFR 140 GFAS, Rennes, France). Hybridized slides were washed manually at room temperature for 2 min with a RiboWash solution (twice) and with a $0.1 \times$ SSC solution (one time).

\section{Microarray data analysis}

All fluorescent images from the microarrays were analyzed using the Genepix 4000B scanner and the Genepix Pro software v6.0 (Axon Instruments, Molecular Devices Co., Sunnyvale, California, USA). Raw data were corrected using MADSCAN software (Le Meur et al., 2004). After subtraction of the background fluorescence, a rank-invariant method and a spatial normalization were performed before scaling of the variance within each slide and between all the slides at the same rank. The corrected values of fluorescence were used to perform statistical analysis using the GeneANOVA software (Didier et al., 2002) considering the three following factors: gene (fluorescence level), treatment (insecticide or water), dye (Cy3 or Cy5) and technical replicates (T1; T2 and $\mathrm{C} 1$; $\mathrm{C} 2$ ). Fold-change values over 1 were considered as upregulated gene expression, while fold-changes under 1 as down-regulated.

\section{Gene ontology analysis}

In order to know which biological processes were overrepresented among regulated transcripts, a gene ontology (GO) analysis was performed using the online available software Blast2GO (Conesa et al., 2005). After the identification of the putative roles for each predicted protein, the regulated transcripts were grouped in the three domains of molecular biology: Biological Processes, Molecular Functions and Cellular Components.

\section{Quantitative RT-PCR}

Total RNAs were isolated from three aphids with the RNeasy Plant Mini Kit, resulting in samples containing between 100 and $400 \mathrm{ng} \mu \mathrm{L}^{-1}$ according to Nanodrop quantifications (Nanodrop Technologies, USA). A reverse transcription using AffinityScript QPCR cDNA Synthesis kit (Agilent) was conducted using $1.5 \mu \mathrm{g}$ of total RNA previously treated with DNAse (DNA-free ${ }^{\mathrm{TM}}$ Kit, Ambion, Canada). The cDNA was then diluted 10 times, using $2 \mu \mathrm{L}$ for PCR in $15 \mu \mathrm{L}$ reaction volume containing: $1 \mu \mathrm{L}(10 \mu \mathrm{mol})$ of each primer, $12.25 \mu \mathrm{L}$ of SYBR Green PCR Master Mix (Applied Biosystems), 0.375 $\mu \mathrm{L}$ of Rox Dye (1:500) and $4.374 \mu \mathrm{L}$ of water. All qPCRs assays were performed in triplicate on a Mx3000P QPCR System (Stratagene) under the following conditions: 10 min at $95^{\circ} \mathrm{C}$, followed by 40 cycles of $15 \mathrm{~s}$ at $95{ }^{\circ} \mathrm{C}$, $15 \mathrm{~s}$ at $57^{\circ} \mathrm{C}, 20 \mathrm{~s}$ a $72{ }^{\circ} \mathrm{C}$. To confirm the absence of nonspecific amplicons, a melting curve was performed immediately after the PCR containing a ramp of 65 to $95{ }^{\circ} \mathrm{C}$. The level of expression for six genes was tested. Specific primers were designed for these genes using the 
available online softwares FastPCR v5.4.30 and AmplifX v1.3.7. An annealing temperature of $60{ }^{\circ} \mathrm{C}$, an amplicon size of $80-120 \mathrm{bp}$, and $45 \%-55 \%$ GC content were selected as parameters. PCR efficiency was determined by performing standard curves in triplicate for all primers sets with selected serial dilutions of a standard batch of cDNA. Primers sequences, GeneBank accession numbers and PCR efficiency values are reported in Table 1.

\section{qRT-PCR data analysis}

Quantitative analyses (two biological and three technical replicates) were conducted using the $\mathrm{Ct}$ comparative method (Applied Biosystems User Bulletin nr 2, P/N 4303859) (Livak and Schmittgen, 2001), using glyceraldehyde 3-phosphate dehydrogenase (GADPH) as reference gene for normalization of input cDNA (Wang and $\mathrm{Xu}, 2010$ ). Comparisons for expression fold-changes were performed between treatments (pure insecticide vs. water), at seven time points $(6,12,18,24,30,36$, and $42 \mathrm{~h})$ and within each biological replicate. The qRT-PCR data were analyzed using a $t$-test for independent samples, using 1 as a reference value for no change in gene expression (Silva et al., 2012). The results are expressed as mean \pm standard error (SE) of two biological and three technical replicates.

\section{RESULTS}

\section{MACE mutation}

The MACE mutation was absent from the different genomic sequences of analyzed aphids, indicating that the predominant clone of the tobacco aphid in Chile lacks the main mutation that confer resistance to organophosphates and carbamates.

\section{Microarray and gene ontology analyses}

Following the normalization by MADSCAN software and the statistical analysis by GeneANOVA, 38 transcripts appeared to be significantly regulated $(\mathrm{p}<0.05)$ in response to insecticide treatment: 13 transcripts were found to be up-regulated (including two unknown transcripts) and 25 transcripts were shown to be down-regulated (including eight unknown transcripts) (Table 2). These 28 known regulated mRNAs were then functionally classified using a gene ontology analysis (GO). Fifteen GO terms were associated with sixteen the regulated genes (Table 3). These GO terms were grouped in three Biological Processes: reproduction (three terms), metabolism (six) and cellular proliferation (six) (Table 3).

Three up-regulated genes: Acyl-CoA-binding protein (Acyl CoA BP) (Fold-change (FC): 1.57), glutathione S-transferase (GST) (FC: 1.30) and dihydrolipoyllysineresidue acetyltransferase (PDC-E2) (FC: 1.28), and three down-regulated genes: heat shock protein (HSP) (FC: 0.74), ubiquinone NADH dehydrogenase (UBIQ) (FC: $0.77)$ and hypothetical protein RNA binding protein (RNBP) (FC: 0.69), were selected to quantify their relative expression (see marked genes in Table 3) using new independent biological replicates. These genes were selected based on their putative participation in a metabolic response to xenobiotics (Figueroa et al., 2007).

\section{Quantification of the expression levels}

The three selected up-regulated RNAs GST, Acyl CoA $\mathrm{BP}$, and PDC-E2 showed an increasing positive foldchange value during the first half of the time-course of response to insecticide with a peak of expression at 24 $\mathrm{h}$ after insecticide pure active ingredient exposure, and decreasing levels of gene expression at later time points (Figure 1). For the selected down-regulated RNAs, UBIC and HSP, we observed lower levels of expression at $24 \mathrm{~h}$ after the exposure to the insecticide. RNABP exhibited a lower level of gene expression at $18 \mathrm{~h}$ after insecticide exposure. The three down regulated RNAs HSP, UBIC and RNABP, showed an increase in expression until they reach $42 \mathrm{~h}$ after insecticide treatment.

Some differences between results obtained in the microarray and qRT-PCR assays were observed. For instance, the UBIQ was down-regulated at $12 \mathrm{~h}$ in the microarray, while in qRT-PCR was observed up-regulated. Similarly, the Acyl CoA BP gene, which was shown to be up-regulated in the microarray at $12 \mathrm{~h}$, was noted downregulated in the $\mathrm{qPCR}$.

Table 1. Transcripts selected for qRT-PCR analysis. Primers sequences, GeneBank accession numbers, PCR efficiency values and $\mathbf{R}^{2}$ standard curves values of the selected genes. GADPH was used as reference gene.

\begin{tabular}{|c|c|c|c|c|}
\hline Gene name & $\begin{array}{c}\text { GeneBank } \\
\text { accession number }\end{array}$ & Primer sequence (5'-3') & $\mathrm{R}^{2}$ & $\begin{array}{c}\text { PCR efficiency } \\
(\%)\end{array}$ \\
\hline \multirow[t]{2}{*}{ Acyl CoA-BP } & EE262188.1 & For: CACAAGCAACGATGAGGCTA & 0.999 & 98.3 \\
\hline & & Rev: GCCATTCGGTTTAAGCTTTG & & \\
\hline \multirow[t]{2}{*}{ GST } & DW361988.1 & For: CTATGGGCGAACCATGAGAT & 0.998 & 91.3 \\
\hline & & Rev: AATGGCATGGTAGGCTTGAG & & \\
\hline \multirow{2}{*}{ PDC-E2 } & EE261058.1 & For: GAGGAAACACCCCAGGAATTGA & 0.998 & 94.9 \\
\hline & & Rev: TTGTCTCCTGGTAGCTTATGCC & & \\
\hline \multirow[t]{2}{*}{ NADH } & EE262674.1 & For: TTGACAACAGAGAGCGTTGG & 0.999 & 91.7 \\
\hline & & Rev: GGCATCAGCTCTGTCAACAA & & \\
\hline \multirow[t]{2}{*}{ HSP } & EE570079.1 & For: AGCATCAACTCTCCGCTGAT & 0.997 & 92.9 \\
\hline & & Rev: ACCGTCTTGACGACGATTTC & & \\
\hline \multirow[t]{2}{*}{ RNABP } & EE262993.1 & For: GGCATAGAAATGGTCCTTGGCT & 0.996 & 93.9 \\
\hline & & Rev: GGGAAGTCAAACCAGGACACAT & & \\
\hline \multirow[t]{2}{*}{ GADPH } & DW011095.1 & For: TGGCTTTCAGAGTACCAGTTGC & 0.998 & 99.3 \\
\hline & & Rev: CTTCAGCGGCTTCCTTGACTTT & & \\
\hline
\end{tabular}

GADPH: Glyceraldehyde 3-phosphate dehydrogenase. 
Table 2. List of differentially expressed transcripts detected by microarray experiments. Transcripts significantly regulated in the microarray are enlisted with their respective information (GeneBank accession number, fold change value in the microarray) and sequence blast results (species, protein, and E-value).

\begin{tabular}{|c|c|c|c|c|}
\hline $\begin{array}{l}\text { Accession number } \\
\text { of EST }\end{array}$ & $\begin{array}{l}\text { Fold change } \\
\text { value }\end{array}$ & Species & Protein & E Value \\
\hline ID0AAK4YG14* & 1.57 & Acyrthosiphum pisum & acyl-CoA-binding protein isoform 2 & $2,00 \mathrm{E}-42$ \\
\hline ID0AFF2DD01 & 1.30 & A.pisum & similar to AGAP000679-PA, partial length $=373$ & $3,00 \mathrm{E}-95$ \\
\hline MpW-IV-F01 & 1.30 & A.pisum & similar to glutathione S-transferase-like protein isoform 2 & $2,00 \mathrm{E}-18$ \\
\hline ID0AFF14DB $12^{*}$ & 1.28 & A. pisum & $\begin{array}{l}\text { similar to dihydrolipoyllysine-residue acetyltransferase component of pyruvate } \\
\text { dehydrogenase complex, mitochondrial precursor (E2) }\end{array}$ & $9,00 \mathrm{E}-110$ \\
\hline ID0AAK9YO06 & 1.28 & A.pisum & similar to B-cell leukemia/lymphoma 6 & $1,00 \mathrm{E}-41$ \\
\hline ID0AAK3YP12 & 1.25 & A.pisum & Unknown & \\
\hline ID0AFF11BA02 & 1.24 & A. pisum & Unknown & \\
\hline ID0AFF1DD09 & 1.22 & A.pisum & similar to lethal (2) k14505 CG8674-PA & $2,00 \mathrm{E}-52$ \\
\hline ID0AFF4AE03 & 1.20 & Tribolium castaneum & similar to N-acetyl-galactosaminyl-transferase & $2,00 \mathrm{E}-78$ \\
\hline ID0AAK3YM01 & 1.19 & A.pisum & similar to Der1-like domain family member 1 & $8,00 \mathrm{E}-93$ \\
\hline ApDT-XXXIII-C2 & 1.18 & A.pisum & Acyrthosiphon pisum strain 5A mitochondrion, complete genome & $0,00 \mathrm{E}+00$ \\
\hline ID0AAK10YH08 & 1.16 & A. pisum & Similar to CG7546 CG7546-PA (LOC100166345), mRNA & $5,00 \mathrm{E}-10$ \\
\hline ID0AAK3YL18 & 1.15 & A.pisum & hypothetical protein & $4,00 \mathrm{E}-69$ \\
\hline ID0AFF10DF02 & 0.87 & A.pisum & similar to ribosomal protein S27A & $1,00 \mathrm{E}-59$ \\
\hline ID0AFF5CG08 & 0.86 & A.pisum & Unknown & $1,00 \mathrm{E}-06$ \\
\hline ID0AAK4YG24 & 0.84 & A. pisum & Unknown & 0.16 \\
\hline ID0AAK2YN05 & 0.84 & A.pisum & similar to phosducin-like 3 , partial & $3,00 \mathrm{E}-68$ \\
\hline ID0AAK6YB03 & 0.85 & A.pisum & Unknown & $1,00 \mathrm{E}-16$ \\
\hline ID0AAK8YM07 & 0.83 & A.pisum & hypothetical protein LOC100160539 & $3,00 \mathrm{E}-44$ \\
\hline ID0AAK8YE17 & 0.83 & A.pisum & hypothetical protein LOC100160953 & $5,00 \mathrm{E}-80$ \\
\hline ID0AAK6YN06 & 0.82 & Apis mellifera & similar to GTPase activating protein and VPS9 domains 1 isoform 1 & $2,00 \mathrm{E}-15$ \\
\hline ID0AFF8BH01 & 0.82 & A.pisum & similar to mitochondrial ribosomal protein L55 CG14283-PA & $2,00 \mathrm{E}-37$ \\
\hline ApDT-XXXIV-H7 & 0.81 & A.pisum & Unknown & 1.5 \\
\hline ID0AFF4AB 10 & 0.81 & A.pisum & Unknown & 2.2 \\
\hline ApHL3LD-III-B11 & 0.80 & A. pisum & Unknown & 0.088 \\
\hline ApHL3LD-XVIII-G2 & 0.80 & A. pisum & Unknown & 0.24 \\
\hline ID0AAK2YB12 & 0.80 & A.pisum & dephospho-CoA kinase & $8,00 \mathrm{E}-24$ \\
\hline ID0AAG15BB04 & 0.79 & A.pisum & 16S large subunit ribosomal RNA & $0,00 \mathrm{E}+00$ \\
\hline ID0AAG10AG10 & 0.79 & A.pisum & similar to internalin A, putative & $2,00 \mathrm{E}-100$ \\
\hline ID0AFF13AH04 & 0.78 & A. mellifera & similar to spectrin alpha chain & $8,00 \mathrm{E}-90$ \\
\hline ID0AFF13DD04 & 0.78 & A.pisum & similar to CG5800 CG5800-PA & $1,00 \mathrm{E}-78$ \\
\hline ID0AAG11CA03* & 0.78 & A.pisum & NADH dehydrogenase (ubiquinone) $\mathrm{Fe}-\mathrm{S}$ protein 4 & $2,00 \mathrm{E}-109$ \\
\hline ID0AAK8YE09 & 0.76 & A.pisum & similar to TATA-binding protein, putative & $7,00 \mathrm{E}-112$ \\
\hline ID0AAK8YB01 & 0.75 & A. pisum & similar to spectrin & $1,00 \mathrm{E}-72$ \\
\hline ID0AAK1YJ21* & 0.74 & A. pisum & similar to heat shock protein hsp21.4 & $4,00 \mathrm{E}-79$ \\
\hline ID0AFF3CE04 & 0.71 & A. pisum & similar to insulin receptor & $3,00 \mathrm{E}-18$ \\
\hline ID0AAK2YH19* & 0.69 & A. pisum & similar to AGAP003344-PA (Acypi007529); hypothetical RNABP LOC100166675 & $4,00 \mathrm{E}-79$ \\
\hline ID0AAK6YB12 & 0.57 & A.pisum & Unknown & 1.5 \\
\hline
\end{tabular}

* Genes selected for relative expression quantification.

\section{DISCUSSION}

Table 3. Gene ontology (GO) annotation of differentially expressed transcripts. Enriched GO terms with their respective identities and function according to GO analysis using Blast2go software are reported.

\begin{tabular}{lll}
\hline GO ID & \multicolumn{1}{c}{ Function } & GO terms \\
\hline GO:0006412 & translation & Metabolism \\
GO:0055114 & oxidation reduction & Metabolism \\
GO:0040010 & positive regulation of growth rate & Reproduction \\
GO:0009408 & response to heat & Metabolism \\
GO:0006310 & DNA recombination & Cell proliferation \\
GO:0007283 & spermatogenesis & Reproduction \\
GO:0015937 & coenzyme A biosynthetic process & Metabolism \\
GO:0030111 & regulation of Wnt receptor signaling pathway & Reproduction \\
GO:0051301 & cell division & Cell proliferation \\
GO:0006357 & regulation of transcription from RNA & Cell proliferation \\
& polymerase II promoter & \\
GO:0007067 & mitosis & Cell proliferation \\
GO:0016568 & chromatin modification & Cell proliferation \\
GO:0043461 & proton-transporting ATP synthase complex & Metabolism \\
& assembly & \\
GO:0006464 & protein modification process & Metabolism \\
GO:0042127 & regulation of cell proliferation & Cell proliferation \\
\hline
\end{tabular}

The M. persicae nicotianae genotype used in this study was initially classified as carboxylesterase R1 and $k d r$ sensitive (Fuentes-Contreras et al., 2004), and the results presented here indicate this genotype is MACE susceptible due to the lack of the insensitivity mutation associated with resistance to organophosphates and carbamates (Anstead et al., 2008). Considering that MACE is the main mechanism reported to explain resistance against carbamate insecticides, the prevalence of this $M$. persicae nicotianae clone in regions where carbamates are frequently used is intriguing, particularly because this putative superclone is widely geographic distributed (Zepeda-Paulo et al., 2010). Hence, other nonmutational mechanisms of insecticide resistance should explain the time-persisting prevalence and frequency of this aphid clone on insecticide sprayed tobacco crops (Criniti et al., 2008).

Hence, the transcriptomic approach performed here explored for other putative insecticide resistance mechanisms involved in the prevalence of this clone in the field, allowing the identification of 38 transcripts 

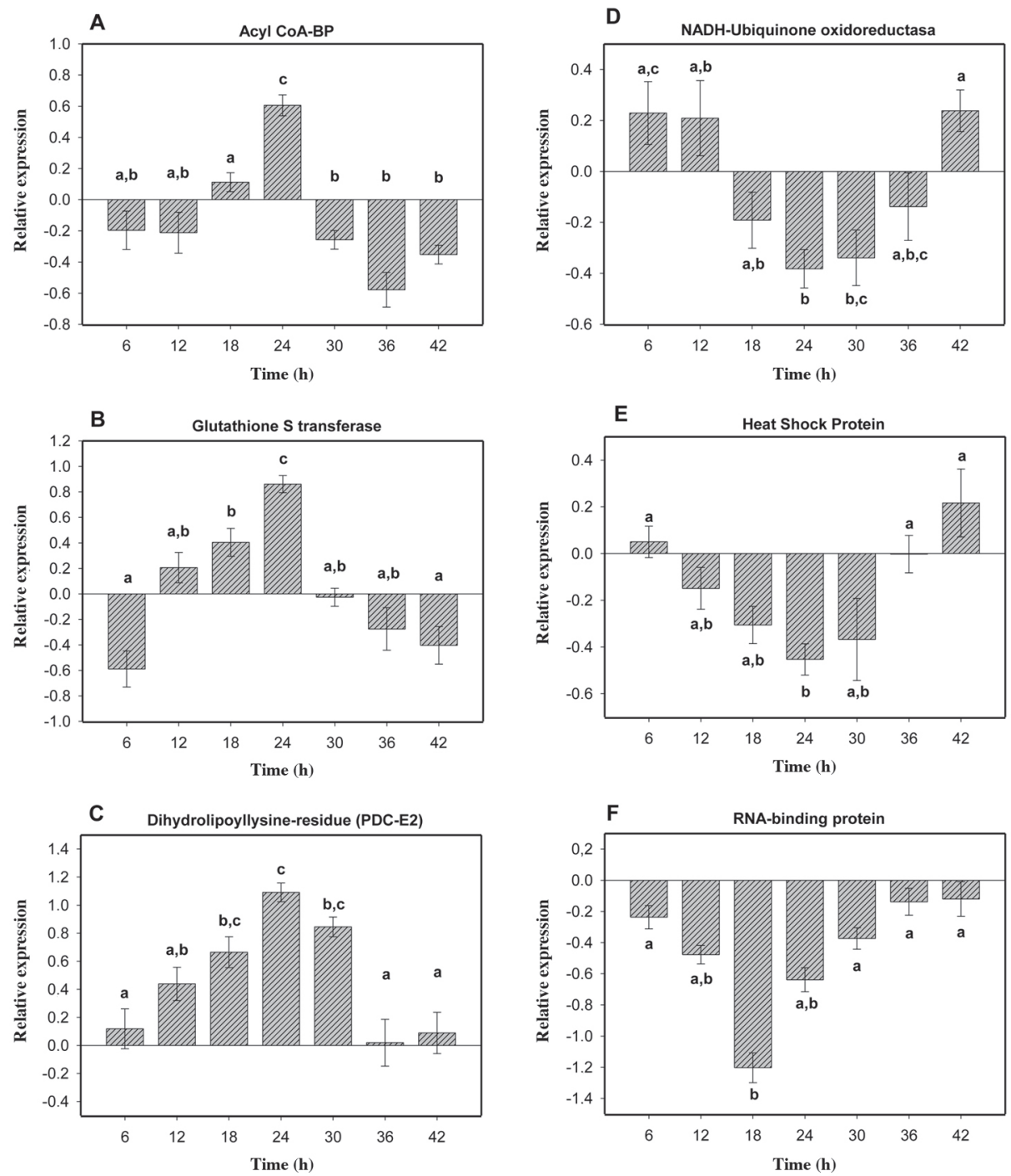

Each bar shows the mean $\pm \mathrm{SE}$ of two biological replicates. Letters indicate significant differences among bars $(t$-test, $P<0.05)$.

Figure 1. Time-course analysis of gene expression levels following insecticide application. Each panel shows the relative gene expression level determined by real time PCR at different time points after tobacco aphids were sprayed with carbamate insecticide pure active ingredient.

differentially expressed after exposing the tobacco aphid to a carbamate insecticide (13 up-regulated and 25 downregulated genes, including 10 unknown genes). The GO terms associated with these 38 regulated genes indicate that most of them are grouped into metabolic processes, suggesting a possible metabolic insecticide resistance pathways involved, such as xenobiotic detoxification related enzymes (Figueroa et al., 2007; Li et al., 2007).

Among those differentially expressed transcripts, we selected three up-regulated genes that are putatively involved in xenophobic responses. The Acyl-CoA-binding protein (EC 3.4.17.3), also known as a diazepam-binding inhibitor (DBI), is involved in benzodiazepine receptor modulation, acyl CoA metabolism, steroidogenesis, insulin secretion, and drug dependence (Rasmussen et al., 1994). This transcript has been shown to be upregulated by DDT insecticide in Drosophila resistant genotypes (Pedra et al., 2004), which suggests a putative role in insecticide resistance for this gene also in the case of aphids. The second over-expressed RNA we selected was glutathione S transferase (GST, EC 2.5.1.18), which is a phase II enzyme of xenobiotic detoxification. In aphids, the GST contributes to tolerance to secondary metabolites from their host plants and has been implicated in resistance to carbamate, pirimicarb and cyclodiene endosulfan insecticides (Francis et al., 2005; Perera et al., 2008). The third transcript selected corresponds to adihydrolipoyllysine-residue acetyltransferase (PDC-E2) (EC 1.8.1.4), which is present in the mitochondria and plays a central role in the energetic metabolism. PDC-E2 
links the glycolysis to the citric acid cycle, resulting in the production of several molecules such as NADH, which plays a key role during the mitochondrial respiratory chain for ATP production. Deficits in PDC-E2 result in a lactic acidosis due to metabolization of pyruvate into lactic acid (Yu et al., 2008). Carbamate insecticide applications usually result in an uncontrollable muscular contraction in target insects, which significantly increases the energetic recruitments, causing a high pyruvate concentration and producing a "bottle neck" between glycolysis and citric acid cycle that trigger a severe lactic acidosis (Fukuto, 1990). The increased levels of PDC-E2 transcripts observed in our experiments suggests a molecular strategy tending to avoid this "bottle neck" between these metabolic processes, and thereby to prevent the lactic acidosis induced by carbamate insecticide in the MACEsusceptible tobacco aphid clone studied.

Regarding the three down-regulated genes selected for qPCR validation, the UBIQ (EC 1.6.5.3) participates in the electron transport complex of the mitochondrial respiratory chain complex I that use NADH as substrate. This is an essential molecule for several physiological processes as muscular contraction playing a key role during the ATP synthesis (Friedrich et al., 2000), and the down-regulation observed after insecticide exposure could be result in uncontrollable muscular contractions prevention by reducing the ATP availability. Other selected down-regulated gene was a heat shock protein (HSP). The HSPs belong to a family of proteins participating in numerous cellular processes (i.e., protein sorting, signal transduction, gene expression regulation by post-transcriptional control of RNAs, DNA replication, and protein synthesis) (Johnstone and Lasko, 2001). These proteins are usually produced in response to a variety of biotic and abiotic stresses (Picard, 2002), as well as in response to insecticides, showing inter specific variations in the expression patterns; in some cases the HSPs are increased under carbamate exposure in the brown planthopper (Nilaparvata lugens) (Sharma et al., 2004), or show an invariable expression when the cabbage armyworm (Mamestra brassicae) is exposed to permethrin, chlorfluazuron, chlorfenapyr, prothiofos, methomyl, and thiocyclam (Sonoda and Tsumuki, 2007), thus suggesting a non-specific response of HSPs to insecticides. Finally, the RNABP were found also to be down-regulated. This gene belongs to a family of genes coding for proteins that play key roles during gene expression regulation by post-transcriptional control of RNAs (Wickens et al., 2000). Thus, the down regulation of RNABP transcripts exhibited after aphids exposure to carbamate insecticide suggest a decreased gene expression regulation at RNA post-transcriptional level, which could favor a gene expression regulation at other levels of regulation (e.g., DNA transcription, protein translation and protein posttranslational mechanisms) (Wickens et al., 2000).
The different results obtained in the microarray and qRT-PCR assays (e.g., RNABP and Acyl coA at $12 \mathrm{~h}$ ) are possibly due to the fact that a commercial insecticide was used in the microarray experiments, while the insecticide pure active ingredient was used in the qPCR assays. Additionally, the sensitivity of microarray and qPCR assays is different and can also explain those differences, since the microarray is a qualitative while qPCR is a quantitative technique.

The results reported here are in accordance to the insecticide residual effect time observed in the field (12-24 h) (Estay, 2006), because it matches with the time-course of aphid's gene expression modification in response to insecticide. This suggests that aphids could moderate the effects of certain insecticides like carbamates by using the cholesterol and pyruvate metabolism to prevent lactic acidosis, while using a GST detoxification system to detoxify the organism, thus decreasing the gene expression when the presence of the active insecticide is lower. In the opposite side, HSP and RNABP remain down-regulated after insecticide application, apparently for deviating energy use for other metabolic pathways. The downregulation of NADH ubiquinone dehydrogenase could be preventing uncontrollable muscular contractions produced by carbamate insecticide in a sensitive aphid clone by reducing the ATP availability, recovering their expression levels when the presence of the active insecticide is lower.

The results obtained in this work, suggest the involvement of resistance mechanisms other than those previously reported for M. persicae (Silva et al., 2012), thus complementing our picture of the genes underlying insecticide resistance in aphids. Further functional studies including proteomics, RNAi and biochemical studies will help going deeper into the characterization of the insecticide resistance mechanisms that participate in aphids, which should explain the persistence of certain aphid clones not carrying insensitivity mutations under strong insecticide applications in the field.

\section{CONCLUSIONS}

The findings reported here show the presence of transcriptomic mechanisms involved in the aphid insecticide resistance by suppression of the insecticides metabolic effects, which could explain the wide geographic distribution exhibited by this superclone in The Americas, including areas where the insecticides are frequently used. This should lead to new hypotheses in order to understand the molecular mechanism underlying the aphid insecticide resistance.

\section{ACKNOWLEDGEMENTS}

PBCT-Anillos ACT38 and FONDECYT 1090378 grants to CCF and DID-UACh grant to MCB. This work is part of the research activities of the Millennium Nucleus Center 
in Molecular Ecology and Evolutionary Applications in the Agroecosystems financed by Iniciativa Científica Milenio grant NC120027.

\section{LITERATURE CITED}

Anstead, J.A., M.S. Williamson, and I. Denholm. 2008. New methods for the detection of insecticide resistant Myzus persicae in U.K. suction trap network. Agricultural and Forest Entomology 10:291-295.

Bass, C., M. Puinean, M. Andrews, P. Cutler, M. Daniels, V.L. Paul, et al. 2011. Mutation of a nicotinic acetylcholine receptor $\beta$ subunit is associated with field evolved resistance to neonicotinoid insecticides in the aphid Myzus persicae. BMC Neuroscience 12:51

Blackman, R.L., and V.F. Eastop. 2000. Aphids on the world's crops: An identification and information guide. $2^{\text {nd }}$ ed. 476 p. Wiley, Chichester, England.

Chowdhury, M.A., S. Banik, B. Uddin, M. Moniruzzaman, N. Karim, and S.H. Gan. 2012. Organophosphorus and carbamate pesticide residues detected in water samples collected from paddy and vegetable fields of the Savar and Dhamrai Upazilas in Bangladesh. International Journal of Environmental Research and Public Health 9:3318-3329.

Conesa, A., S. Götz, J.M. García-Gómez, J. Terol, M. Talón, and M. Robles. 2005. Blast2GO: A universal tool for annotation, visualization and analysis in functional genomics research. Bioinformatics 21:3674-3676.

Criniti, A., E. Mazzoni, S. Cassanelli, P. Cravedi, A. Tondelli, D. Bizzaro, et al. 2008. Biochemical and molecular diagnosis of insecticide resistance conferred by esterase, MACE, $k d r$ and super-kdr based mechanisms in Italian strains of the peach potato aphid, Myzus persicae (Sulzer). Pesticide Biochemistry and Physiology 90:168-174.

Didier, G.,P. Brézellec, E. Remy, and A.Hénaut. 2002. GeneANOVAgene expression analysis of variance. Bioinformatics 18:490-491.

Estay, P. 2006. Catálogo de pesticidas II. Instituto de Investigaciones Agropecuarias (INIA), La Platina, Santiago, Chile. Available at http://platina.inia.cl/entomologia/polinizadores/Catalogo_ Pesticidas_II.pdf (accessed June 2013).

Ffrench-Constant, R.H., P.J. Daborn, and G. Le Goff. 2004. The genetics and genomics of insecticide resistance. Trends in Genetics 20:163-170.

Figueroa, C.C., N. Prunier-Leterme, C. Rispe, F. Sepúlveda, E. Fuentes-Contreras, B. Sabater-Muñoz, et al. 2007. Annotated expressed sequence tags and xenobiotic detoxification in the aphid Myzus persicae (Sulzer). Insect Science 1429-1445.

Francis, F., N. Vanhaelen, and E. Haubruge. 2005. Glutathione S-transferases in the adaptation to plant secondary metabolites in the Myzus persicae aphid. Archives of Insect Biochemistry and Physiology 58:166-174.

Friedrich, T., B. Brors, P. Hellwig, L. Kintscher, T. Rasmussen, D. Scheide, et al. 2000. Characterization of two novel redox groups in the respiratory NADH:ubiquinone oxidoreductase (complex I). Journals of Biochimica et Biophysica Acta 15:1459(2-3):305-309.

Fuentes-Contreras, E., C.C. Figueroa, M. Reyes, L.M. Briones, and H.N. Niemeyer. 2004. Genetic diversity and insecticide resistance of Myzus persicae (Hemiptera: Aphididae) populations from tobacco in Chile: evidence for the existence of a single predominant clone. Bulletin of Entomological Research 94:11-18.

Fukuto, T.R. 1990. Mechanism of action of organophosphorus and carbamate insecticides. Environmental Health Perspectives 87:245-254.

Johnstone, O., and P. Lasko. 2001. Translational regulation and RNA localization in Drosophila oocytes and embryos. Annual Review of Genetics 35:365-406.
Le Meur, N., G. Lamirault, A. Bihouée, M. Steenman, H. BédrineFerran, R. Teusan, et al. 2004. A dynamic, web accessible resource to process raw microarray scan data into consolidated gene expression values: importance of replication. Nucleic Acids Research 32:5349-5358.

Le Trionnaire, G., F. Francis, S. Jaubert-Possamai, J. Bonhomme, E. De Pauw, J.P. Gauthier, et al. 2009. Transcriptomic and proteomic analyses of seasonal photoperiodism in the pea aphid. BMC Genomics 10:456.

Li, X.C., M.A. Schuler, and M.R. Berenbaum. 2007. Molecular mechanisms of metabolic resistance to synthetic and natural xenobiotics. Annual Review of Entomology 52:231-253.

Livak, K.J. 1999. Allelic discrimination using fluorogenic probes and the 5' nuclease assay. Genetic Analysis: Biomolecular Engineering 14(5-6):143-149.

Livak, K.J., and T.D. Schmittgen. 2001. Analysis of relative gene expression data using real-time quantitative PCR and the 2(T) (-Delta Delta C) method. Methods 25:402-408.

Margaritopoulos, J.T., J.A. Tsitsipis, E. Zintzaras, and R.L. Blackman. 2000. Host-correlated morphological variation of Myzus persicae (Hemiptera: Aphididae) populations in Greece. Bulletin of Entomological Research 90:233-244.

Moury, B., F. Fabre, and R. Senoussi. 2007. Estimation of the number of virus particles transmitted by an insect vector. Proceedings of the National Academy of Sciences of the United States of America 104:17891-17896.

Nabeshima, T., T. Kozaki, T. Tomita, and Y. Kono. 2003. An amino acid substitution on the second acetylcholinesterase in the pirimicarb-resistant strains of the peach potato aphid, Myzus persicae. Biochemical and Biophysical Research Communications 307:15-22.

Pedra, J.H., L.M. McIntyre, M.E. Scharf, and B.R. Pittendrigh. 2004. Genome-wide transcription profile of field- and laboratoryselected dichlorodiphenyltrichloroethane (DDT)-resistant Drosophila. Proceedings of the National Academy of Sciences of the United States of America 101:7034-7039.

Perera, M.D., J. Hemingway, and S.P. Karunaratne. 2008. Multiple insecticide resistance mechanisms involving metabolic changes and insensitive target sites selected in anopheline vectors of malaria in Sri Lanka. Malaria Journal 7:168.

Picard, D. 2002. Heat-shock protein 90, a chaperone for folding and regulation. Cell Molecular Life Science 59:1640-1648.

Puinean, A.M., S.P. Foster, L. Oliphant, I. Denholm, L.M. Field, N.S. Millar, et al. 2010. Amplification of a cytochrome P450 gene is associated with resistance to neonicotinoid insecticides in the aphid Myzus persicae. PLoS Genetics 6(6):e1000999.

Rasmussen, J.T., N.J. Faergeman, K, Kristiansen, and J. Knudsen. 1994. Acyl-CoA-binding protein (ACBP) can mediate intermembrane acyl-CoA transport and donate acyl-CoA for beta-oxidation and glycerolipid synthesis. Biochemical Journal 299:165-170.

Raymond, M., C. Chevillon, T. Guillemaud, T. Lenormand, and N. Pasteur. 1998. An overview of the evolution of overproduced esterases in the mosquito Culex pipiens. Philosophical Transactions of the Royal Society of London Series B-Biological Sciences 353:1707-1711.

Sharma, R., S. Komatsu, and H. Noda. 2004. Proteomic analysis of brown planthopper: application to the study of carbamate toxicity. Insect Biochemistry and Molecular Biology 34:425-432.

Silva, A.X., G. Jander, H. Samaniego, J.S. Ramsey, and C.C. Figueroa. 2012. Insecticide resistance mechanisms in the green peach aphid Myzus persicae (Hemiptera: Aphididae) I: A Transcriptomic Survey. PLoS ONE 7(6):e36366. doi:10.1371/ journal.pone.0036366.

Sonoda, S., and H. Tsumuki. 2007. Induction of heat shock protein genes by chlorfenapyr in cultured cells of the cabbage armyworm, Mamestra brassicae. Pesticide Biochemistry and Physiology 89:185-189. 
van-Emden, H., and R. Harrington. 2007. Aphids as crop pests. 58 p. CABI, Wallingford, UK.

Vorburger, C., M. Lancaster, and P. Sunnucks. 2003. Environmentally related patterns of reproductive modes in the aphid Myzus persicae, and the predominance of two "superclones" in Victoria Australia. Molecular Ecology 12:3493-3504.

Wang, G.P., and C.S. Xu. 2010. Reference gene selection for realtime RT-PCR in eight kinds of rat regenerating hepatic cells. Molecular Biotechnology 46:49-57.

Wickens, M., E.B. Goodwin, J. Kimble, S. Strickland, and M.W. Hentze. 2000. Translational control of developmental decisions. Translational control of gene expression. p. 295-370. In Sorenberg, N., J.W.B. Hershey, and M.B. Mathews (eds.) Cold Spring Harbor Laboratory Press, New York, USA.
Yu, S.J. 2008. The toxicology and biochemistry of insecticides. CRC Press, London, England.

Yu, X., Y. Hiromasa, H. Tsen, K. Stoops, E. Roche, and H. Zhou. 2008. Structures of the human pyruvate dehydrogenase complex cores: A highly conserved catalytic center with flexible N-terminal domains. Structure 16:104-114.

Zepeda-Paulo, F.A., J-C. Simon, C.C. Ramírez, E. FuentesContreras, J.T. Margaritopoulos, A.C.C. Wilson, et al. 2010. The invasion route for an insect pest species: The tobacco aphid in the New World. Molecular Ecology 19:4738-4752. 